\title{
Two-photon absorption and broadband optical limiting with bis-donor stilbenes
}

\author{
J. E. Ehrlich, X. L. Wu, I.-Y. S. Lee, Z.-Y. Hu, H. Röckel, S. R. Marder, * and J. W. Perry* \\ Jet Propulsion Laboratory and Beckman Institute, California Institute of Technology, Pasadena, California 91109
}

Received August 12, 1997

\begin{abstract}
Large two-photon absorptivities are reported for symmetrical bis-donor stilbene derivatives with dialkylamino or diphenylamino groups. These molecules exhibit strong optical limiting of nanosecond pulses over a broad spectral range in the visible. Relative to bis(di- $n$-butylamino)stilbene, bis(diphenylamino)stilbene exhibits a $90-\mathrm{nm}$ red shift of its optical limiting band but only a minimal shift of $\sim 13 \mathrm{~nm}$ of its lowest one-photon electronic absorption band. Mixtures of these compounds offer an unprecedented combination of broad optical limiting bandwidth and high linear transparency. (c) 1997 Optical Society of America
\end{abstract}

Materials that exhibit strong nonlinear absorption are currently of considerable interest for a variety of applications, including optical limiting. ${ }^{1-3}$ Such materials can be used in eye and sensor protection applications, optical pulse shaping and processing, and laser mode locking, for example. Much of the research on materials for optical limiting over the past 10 years has centered on sequential single-photon absorptive processes (reverse saturable absorption) in molecules. However, the linear transmission for a material composed of reverse saturable absorbers is necessarily significantly less than unity. Two-photon absorption offers the advantage of high transmission at low incident intensity for fundamental optical frequencies well below the bandgap frequency. However, most known molecular two-photon absorptivities are too small for use in optical limiting applications involving nanosecond pulses. Large two-photon absorptivities were reported recently by He et al. ${ }^{4-6}$ for several organic chromophores, and they reported optical limiting of nanosecond pulses at $1064 \mathrm{~nm}$ for a dialkylamino stilbazolium iodide.

As part of our effort to understand relationships between molecular structure and two-photon absorptivity and to identify advanced materials for optical limiting, we examined a series of symmetrically substituted stilbene derivatives bearing two electron-donor groups. We report that 4,4'-bis(di- $n$-butylamino)stilbene (BDBAS) and 4,4'-bis(diphenylamino)stilbene (BDPAS) exhibit strong two-photon-induced absorption at visible wavelengths as measured with nanosecond pulses. We demonstrate strong optical limiting of visible, nanosecond pulses with these compounds in an $f / 5$ optical system. Moreover, we show that replacement of the di- $n$-butylamino groups with diphenylamino groups results in a 90-nm redshift of the two-photon absorption but in only a $13-\mathrm{nm}$ shift of the onephoton absorption edge, leading to mixtures capable of broadband nanosecond optical limiting with high transparency.

The UV-visible absorption spectra and molecular structures of BDBAS and BDPAS are shown in Fig. 1. BDBAS shows a peak of its one-photon absorption at $374 \mathrm{~nm}$, whereas for BDPAS the peak is redshifted by 13 to $387 \mathrm{~nm}$. Both compounds form so- lutions that are highly transparent across most of the visible spectrum and exhibit a linear transmission of $>90 \%$ at wavelengths of $\geq 500 \mathrm{~nm}$ for a concentration of $0.1 \mathrm{M}$.

Two-photon absorptivities of BDBAS and BDPAS were measured by direct nonlinear optical transmission (NLT), wherein the transmission $T$ of a sample is measured as a function of incident intensity $I_{0}$. Measurements were made on 1-cm path-length solutions in toluene or acetone by use of a collimated laser beam with a cross-sectional area of $0.0025 \mathrm{~cm}^{2}$. A Nd:YAGpumped dye laser with a pulse duration of $\sim 5 \mathrm{~ns}$ and a relatively uniform top-hat spatial profile was employed in these experiments. The incident laser intensity was controlled by neutral-density filters and a half-wave plate-polarizer combination. A beam splitter was used to provide an intensity reference beam. The pulses transmitted by the sample and the reference pulses were detected by 1-cm-diameter silicon photodiodes.

We analyzed the data by performing linear fits of $1 / T$ versus $I_{0}$ to the standard expression ${ }^{7}$ for a pure two-photon absorption process:

$$
1 / T=1+\beta g^{(2)} I_{0} L,
$$

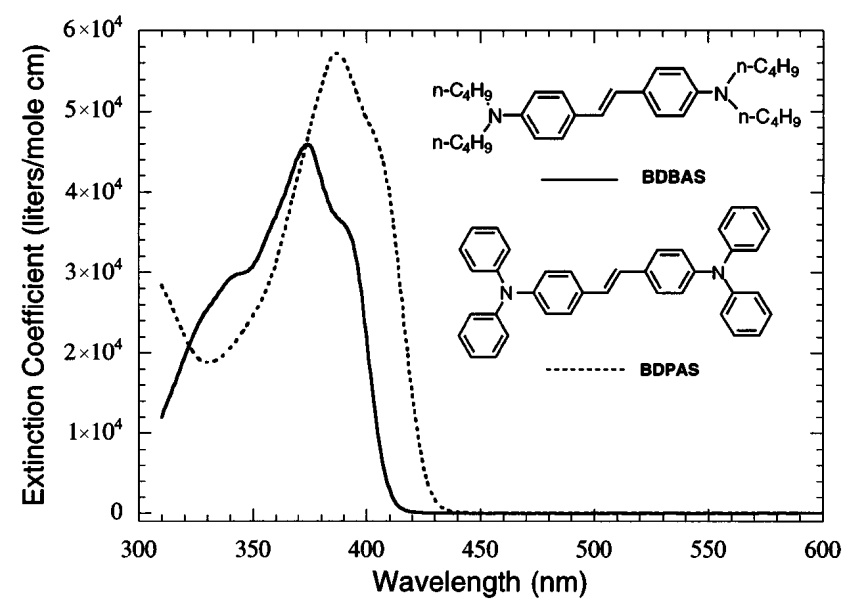

Fig. 1. UV-visible absorption spectra in toluene and molecular structures of BDBAS and BDPAS. 
where $\beta$ is the two-photon absorption coefficient, $g^{(2)}$ is the degree of second-order coherence of the laser pulses, and $L$ is the sample path length. This analysis is appropriate because the measurements reported here were performed in the transparent spectral regions of the compounds where the linear absorption is negligible. Inasmuch as our pulses are not top-hat shaped in time, the two-photon absorptivity we obtain with Eq. (1) should be viewed as a time average over the pulse shape. From the analysis we derive a value for $\beta g^{(2)}$. The $\beta$ values that we report here implicitly contain the factor $g^{(2)}$. The molecular two-photon absorptivity $\delta$ is calculated from the two-photon absorption coefficient with

$$
\delta=\frac{1000 h \nu \beta}{N_{A} C},
$$

where $N_{A}$ is Avogadro's number, $C$ is the sample concentration, and $h \nu$ is the photon energy. To the extent that there may be contributions from higher-order nonlinearities to the observed nonlinear absorption, the two-photon absorptivities reported here should be viewed as effective parameters. The precision in the measurement of the effective two-photon absorption coefficient in our apparatus is $\pm 10 \%$ plus a noise floor of $0.1 \mathrm{~cm} / \mathrm{GW}$, whereas the uncertainty is $\pm 30 \%$.

Both BDBAS and BDPAS exhibit very large twophoton absorptivities as determined by use of nanosecond pulses. Figure 2 shows typical NLT data for BDBAS in acetone solution. At $600 \mathrm{~nm}$, the wavelength of the two-photon absorption peak, we obtain $\beta_{\text {eff }}=3.2 \mathrm{~cm} / \mathrm{GW}$ and $\delta_{\text {eff }}=17700 \times$ $10^{-50} \mathrm{~cm}^{4} \mathrm{~s} /$ photon. Measurements of BDBAS in toluene give $\delta_{\text {eff }}=9300 \times 10^{-50} \mathrm{~cm}^{4} \mathrm{~s} /$ photon, half of the value in acetone, indicating a significant solvent effect. For BDPAS in toluene solution we obtain a peak $\delta_{\text {eff }}$ value of $13000 \times 10^{-50} \mathrm{~cm}^{4} \mathrm{~s} /$ photon at $700 \mathrm{~nm}$. We measured $\beta_{\text {eff }}$ as a function of concentration for a compound closely related to BDBAS, i.e., 4 -di- $n$-butylamino, 4 '-dimethylamino stilbene, and $\beta_{\text {eff }}$ scaled linearly with concentration from 0.05 to $0.3 \mathrm{M}$.

The substitution of the stilbene backbone with strong electron-donating groups plays a major role in determining the position and strength of the nonlinear absorption. Measurements in our apparatus on transstilbene from 560 to $700 \mathrm{~nm}$ showed no measurable nonlinear absorption, corresponding to an upper bound on $\delta_{\text {eff }}$ of $\sim 300 \times 10^{-50} \mathrm{~cm}^{4} \mathrm{~s} /$ photon. The value of $\delta$ for trans-stilbene has been reported to be $12 \times$ $10^{-50} \mathrm{~cm}^{4} \mathrm{~s} /$ photon at $514 \mathrm{~nm}$ as determined by coherent anti-Stokes Raman spectroscopy. ${ }^{8}$ Additionally, measurements of 4,4'-bis-donor stilbenes with one di- $n$ butylamino group and an $n$-butyloxy group (a weaker electron donor) as well as a molecule with two $n$ butyloxy groups (for which data at $590 \mathrm{~nm}$ are shown in Fig. 1) exhibit no measurable two-photon absorption from 560 to $700 \mathrm{~nm}$, verifying that two strong donor groups are required for strong two-photon absorption at relatively long wavelengths, i.e., longer than $560 \mathrm{~nm}$.

The two-photon absorptivities for the bis-donor stilbenes observed with nanosecond pulses are unusually large, comparable with the largest ones reported to date for organic molecules. For example, He et al. reported values as determined by NLT measurements of $850-2980 \times 10^{-50} \mathrm{~cm}^{4} \mathrm{~s} /$ photon at $602 \mathrm{~nm}$ for bis(benzthiazole)-di(dodecyloxy)thiophene (BBTDOT) in various media, ${ }^{4,5} 47000 \times 10^{-50} \mathrm{~cm}^{4} \mathrm{~s} /$ photon at $1064 \mathrm{~nm}$ for trans-4-[( $N$-(ethyl), $N$-(2-hydroxyethyl) aminostyryl] $N$-methylpyridinium tetrafluorborate, ${ }^{6}$ and $3800 \times 10^{-50} \mathrm{~cm}^{4} \mathrm{~s} /$ photon at $800 \mathrm{~nm}$ for 4-[N-(2-hydroxyethyl)- $N$-(methyl)amino]-4'-(6-hydroxy hexylsulfonyl)stilbene. ${ }^{9}$ In comparison, values reported for a wide range of organic fluorophores, including Rhodamine B, as obtained by femtosecond two-photon-excited fluorescence methods at wavelengths between 690 and $1050 \mathrm{~nm}$ were below $300 \times 10^{-50} \mathrm{~cm}^{4}$ s/photon. ${ }^{10}$

Whereas pure two-photon absorption is strictly a third-order nonlinear optical process, higher-order contributions to two-photon absorption determinations by NLT and Z-scan methods have been reported for BBTDOT (Ref. 11) as well as for alkylcyanobiphenyl liquidcrystal molecules. ${ }^{12-14}$ The effective fifth-order optical nonlinearity in these measurements has been identified as being due to two-photon-induced excitedstate absorption. We have found that there is a substantial pulse-width dependence of the two-photon absorptivity of BDBAS and BDPAS, with much smaller values, by a factor of 40, obtained for picosecond pulses, consistent with a strong contribution by two-photoninduced excited-state absorption to the nanosecond pulse measurements. Picosecond pump-probe measurements and nanosecond transient absorption measurements, which we intend to report elsewhere, confirm the presence of two-photon-induced excitedstate absorption.

Given the large effective two-photon absorptivity observed for BDBAS and BDPAS, we examined their optical limiting performance, using nanosecond laser pulses and an $f / 5$ optical system. The transmitted laser beam was collected with a matching $f / 5$ lens and directed onto a photodiode detector. The focus of the beam was estimated to be $\sim 5 \mu \mathrm{m}$ in diameter and was located $\sim 2 \mathrm{~mm}$ from the exit window of the $1-\mathrm{cm}$ sample cell. Figure 3 shows the optical limiting

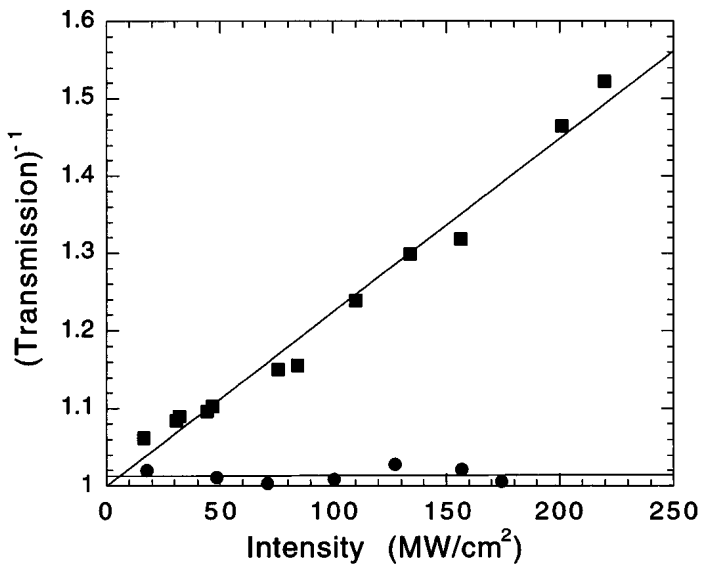

Fig. 2. Intensity-dependent transmission of BDBAS (squares) at $610 \mathrm{~nm}$ and 4,4'-bis(di- $n$-butyloxy)stilbene (circles) at $590 \mathrm{~nm}$ in acetone at $0.01 \mathrm{M}$ with 5 -ns pulses. For BDBAS at $610 \mathrm{~nm}, \delta_{\text {eff }}=12000 \times 10^{-50} \mathrm{~cm}^{4} \mathrm{~s} /$ photon. 


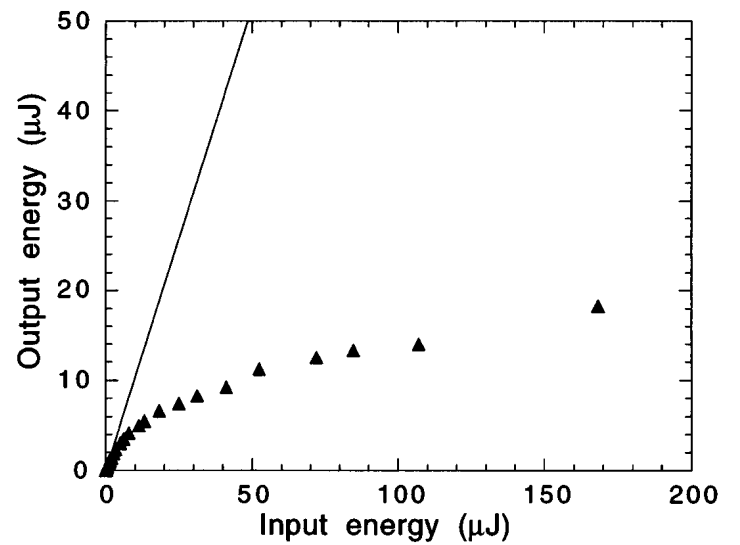

Fig. 3. Optical limiting response of BDBAS in acetone solution at a concentration of $0.14 \mathrm{M}$ for 600-nm, 5-ns laser pulses and an $f / 5$ optical system. The solid line corresponds to the linear transmission of the solution, which was 0.95 .

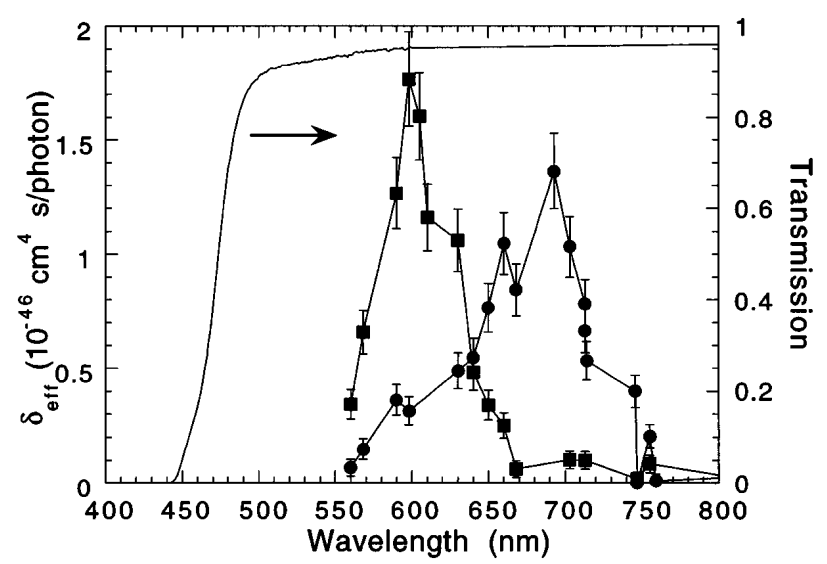

Fig. 4. Spectrum of effective two-photon absorptivity for BDBAS in acetone (squares) and BDPAS in toluene (circles), both at 0.01-M concentrations. Error bars indicate the precision of the measurements. The linear transmission for a toluene solution containing a mixture of both compounds (at 0.1-M concentration) is shown as the solid curve without points.

response of BDBAS for $600-\mathrm{nm}, 5$-ns laser pulses. For a concentration of $0.14 \mathrm{M}$, the strong-signal suppression $(1 / T)$ at an incident energy of $160 \mu \mathrm{J}$ was $\sim 9$, whereas the linear transmission of the sample was 0.95 . Measurement at an incident energy of $1000 \mu \mathrm{J}$ resulted in a suppression of 25 . At high incident energies we observed the formation of a nonlinear refractive ring pattern on the transmitted wave front, which was likely due to thermal and possibly excitedstate refractive-index changes. Although there was certainly a contribution to the observed limiting response from this effect, most of the transmitted energy was collected by the output lens and detected.

Wavelength-dependent measurements of the effective two-photon absorptivity revealed a significant redshift of the peak absorptivity for BDPAS compared with BDBAS. As shown in Fig. 4, the peak for BDBAS occurs at $\sim 600 \mathrm{~nm}$, whereas the band center for BDPAS occurs at $\sim 690 \mathrm{~nm}$. As discussed above, there is only a small redshift of $\sim 13 \mathrm{~nm}$ in the peak of the one-photon absorption on going from BDBAS to BDPAS. Clearly the diphenylamino end groups, compared with the dialkylamino end groups, selectively stabilize the two-photon state relative to the lowest one-photon state. As a consequence, a mixture of BDBAS and BDPAS provides an unprecedented combination of substantial nonlinear absorptivity over a very broad spectral range, from 560 to $730 \mathrm{~nm}$, and a high linear transmission of $>90 \%$ over the same range. We established the additive nature of the two-photon absorptivity by examining solutions of a mixture of BDBAS and BDPAS, each at a concentration of $0.01 \mathrm{M}$ in toluene, and comparing them with solutions of the individual compounds. The absorptivity of the mixture was found to be a linear sum of those for each compound over the range of wavelengths examined $(590-650 \mathrm{~nm})$. Thus, by using a combination of bis-amino stilbenes in the same host medium, one can obtain a broad-bandwidth optical limiting response simultaneously with a high transparency.

This research was performed in part at the Jet Propulsion Laboratory, California Institute of Technology, as part of its Center for Space Microelectronics Technology and was supported by Wright-Patterson Air Force Base. Support at Caltech from the U.S. Office of Naval Research (ONR), the Center for Advanced Multifunctional Nonlinear Optical Polymers and Molecular Assemblies (which is supported by ONR), and the U.S. Air Force Office of Scientific Research, is gratefully acknowledged.

*To whom correspondence should be addressed.

\section{References}

1. J. W. Perry, in Nonlinear Optics of Organic Molecules and Polymers, H. S. Nalwa and S. Miyata, eds. (CRC, Boca Raton, Fla., 1997), p. 813.

2. J. D. Bhawalkar, G. S. He, and P. N. Prasad, Rep. Prog. Phys. 59, 1041 (1996).

3. L. W. Tutt and T. F. Boggess, Prog. Quantum Electron. 17, 299 (1993).

4. G. S. He, G. C. Xu, P. N. Prasad, B. A. Reinhardt, J. C. Bhatt, and A. G. Dillard, Opt. Lett. 20, 435 (1995).

5. G. S. He, R. Gvishi, P. N. Prasad, and B. Reinhardt, Opt. Commun. 117, 133 (1995).

6. G. S. He, J. D. Bhawalkar, C. F. Zhao, and P. N. Prasad, Appl. Phys. Lett. 67, 2433 (1995).

7. R. Loudon, The Quantum Theory of Light (Oxford U. Press, London, 1973).

8. R. J. M. Anderson, G. R. Holtom, and W. M. McClain, J. Chem. Phys. 70, 4310 (1979).

9. J. D. Bhawalkar, G. S. He, C.-K. Park, C. F. Zhao, G. Ruland, and P. N. Prasad, Opt. Commun. 124, 33 (1997).

10. C. Xu and W. W. Webb, J. Opt. Soc. Am. B 13, 481 (1996).

11. A. A. Said, C. Wamsley, D. J. Hagan, E. W. Van Stryland, B. A. Reinhardt, P. Roderer, and A. G. Dillard, Chem. Phys. Lett. 228, 646 (1994).

12. W. Zhao and P. Palffy-Muhoray, Appl. Phys. Lett. 65, 673 (1994).

13. L. Li, G. Hu, P. Palffy-Muhoray, M. A. Lee, and H. J. Yuan, Proc. SPIE 1692, 107 (1992).

14. K. J. McEwan and R. C. Hollins, J. Nonlin. Opt. Phys. Mat. 4, 245 (1995). 